\title{
Perfect reconstruction of signal-a new polynomial matrix inverse approach
}

\author{
Wojciech P. Hunek* (D) and Paweł Majewski
}

\begin{abstract}
The paper outlines a new approach to the signal reconstruction process in multivariable wireless communications tasks. A new solution is proposed using the so-called Smith factorization, which is efficiently used in the synthesis of control systems described by polynomial matrix notation. In particular, the so-called polynomial S-inverse is used, which, together with the applied degrees of freedom, creates a potential for the improvement of the operation of wireless data communications systems comprising different numbers of inputs/antennas and outputs/antennas. Simulations performed in the Matlab environment indicate the practical applicability of the proposed solution.
\end{abstract}

Keywords: Perfect signal reconstruction, Polynomial matrix approach, Polynomial matrix inverses, Non-square MIMO systems, Communications systems

\section{Introduction}

In mobile wireless communications, increasing attention is paid to the quality and quantity of data transmitted in a given unit of time. A higher capacity of the radio channel of MIMO (multi-input/multi-output) systems, i.e., systems comprising multiple inputs/antennas and multiple outputs/antennas, is gradually replacing the traditional SISO (single-input/single-output) approach [1]. This is confirmed by the widely used WiMAX, WiFi 802.11n, DVB-T, or LTE/LTE advanced standards, the majority of which use the OFDM (orthogonal frequency division multiplexing) technology [2-5]. Therefore, an increased capacity of these systems requires the use of a large number of subcarriers and a parallel data transmission mechanism. An intriguing alternative can be therefore seen in systems based on different numbers of transmitting and receiving antennas, where, unlike in the SISO and square MIMO systems (identical numbers of inputs and outputs), the so-called non-uniqueness is present, thus creating viable possibilities of improving the efficiency of the non-square wireless communications systems. It should be emphasized that the drawback observed here in the form of inter-channel interference (ICI) is eliminated by applying the so-called SVD (singular value decomposition) form in the signal reconstruction process [6-8],

*Correspondence: w.hunek@po.opole.pl

Opole University of Technology, Prószkowska 76, 45-758 Opole, Poland dedicated solely to the traditional analysis based on a parameter matrix calculus [9-11]. On the other hand, in the approach based on the polynomial matrix calculus [12-14], the aforementioned dysfunction is eliminated by using the PSVD (polynomial SVD) [15-17]. Unfortunately, all mentioned approaches to the signal perfect reconstruction [18-20], also those including the Smith decomposition method [21], have not so far included the so-called degrees of freedom [22]. Therefore, they were solely related to a certain "optimal" solution associated with the application of the minimum-norm/least-squares inverses to an Eigen matrix obtained from the factorization process [11]. What is important is that even though the above methods involve an infinite number of pairs of precoder-equalizer, our degrees of freedom should be understood in terms of usage of the different inverses to Eigen matrix under a unique precoder-equalizer pair. It will be shown that former cases are quite inappropriate for the polynomial matrix description [16], and the Smith factorization method proposed here outperforms the classical solutions remarkably.

\subsection{Method}

In this paper, a new analytical solution to the signal perfect reconstruction is presented. Not only does this approach in discrete-time domain, dedicated to non-square systems, eliminates parasitic effects in the form of ICI and ISI (inter-symbol interference), but also it efficiently uses 
the highly expected mechanism of non-uniqueness, which considerably improves the "robustness" of wireless communications. The approach proposed herein is based on the so-called Smith form of non-square polynomial matrices, which is the foundation of the polynomial $S$-inverse $[22,23]$.

\section{System representation}

We carry out the analysis of the wireless data communications system with $N_{\mathrm{T}}$-transmitter antennas and $N_{\mathrm{R}}$ receiver antennas described by the (discrete) polynomial matrix in the form of $\underline{\mathbf{C}}\left(z^{-1}\right)[16]$

$$
\underline{\mathbf{C}}_{N_{\mathrm{R}} \times N_{\mathrm{T}}}\left(z^{-1}\right)=\sum_{n=0}^{L_{\mathrm{c}}-1} \underline{\mathbf{c}}_{\mathbf{n}} z^{-n},
$$

where $\left(L_{\mathrm{c}}-1\right)$ is the order of the FIR (finite impulse response) matrix $\underline{\mathrm{C}}\left(z^{-1}\right)$.

The deterministic signal reconstruction process is performed here in accordance with the difference equation

$$
\mathbf{R}(t)=\underline{\mathbf{C}}\left(q^{-1}\right) \mathbf{S}(t),
$$

where the vector of transmitted signals $\mathbf{S}(t)$ and the vector of received signals $\mathbf{R}(t)$ have the dimensions $N_{\mathrm{T}}$ and $N_{\mathrm{R}}$, respectively (see Section 4 ). Note that the symbol $t$ denotes a discrete-time domain, whereas $q^{-1}$ is the backward shift operator corresponding to $z^{-1}$ one.

In the new perfect reconstruction approach presented in this paper, a number of different inverses are used; they are described in detail in the next section.

\section{Inverses of non-square polynomial matrices}

Due to the non-square form of $\underline{\mathbf{C}}_{N_{\mathrm{R}} \times N_{\mathrm{T}}}\left(z^{-1}\right)$, the authors suggest using new inverses of non-square polynomial matrices [22-25] in the signal reconstruction process [22]. We start with the classical minimum-norm right and least-squares left inverses known as $T$-inverses in the polynomial case.

\subsection{T-inverses}

For the polynomial matrix $\underline{\mathbf{C}}\left(q^{-1}\right)=\underline{\mathbf{c}}_{0}+\underline{\mathbf{c}}_{1} q^{-1}+\ldots+$ $\mathbf{c}_{\mathbf{m}} q^{-m}$ of full normal rank, the unique minimum-norm right $T$-inverse is defined as

$$
\underline{\mathbf{C}}_{0}^{\mathrm{R}}\left(q^{-1}\right)=\underline{\mathbf{C}}^{\mathrm{T}}\left(q^{-1}\right)\left[\underline{\mathbf{C}}\left(q^{-1}\right) \underline{\mathbf{C}}^{\mathrm{T}}\left(q^{-1}\right)\right]^{-1},
$$

while the unique least-squares left $T$-inverse is in the following form

$$
\underline{\mathbf{C}}_{0}^{\mathrm{L}}\left(q^{-1}\right)=\left[\underline{\mathbf{C}}^{\mathrm{T}}\left(q^{-1}\right) \underline{\mathbf{C}}\left(q^{-1}\right)\right]^{-1} \underline{\mathbf{C}}^{\mathrm{T}}\left(q^{-1}\right) .
$$

\section{$3.2 \tau$-inverses}

The non-unique right $\tau$-inverse of the polynomial matrix $\underline{\mathbf{C}}\left(q^{-1}\right)$ is defined as $\left(N_{\mathrm{R}}<N_{\mathrm{T}}\right)$

$$
\begin{aligned}
\underline{\mathbf{C}}^{\mathrm{R}}\left(q^{-1}\right)=\{ & \mathbf{I}_{N_{\mathrm{T}}}+\left[\underline{\beta}_{\mathbf{s}}\left(q^{-1}\right)\right]_{0}^{\mathrm{R}}\left[\underline{\mathbf{C}}\left(q^{-1}\right)\right. \\
& \left.\left.-\underline{\beta}\left(q^{-1}\right)\right]\right\}^{-1}\left[\underline{\beta}_{\mathbf{s}}\left(q^{-1}\right)\right]_{0}^{\mathrm{R}},
\end{aligned}
$$

where polynomial matrices $\underline{\beta}\left(q^{-1}\right)$ and $\underline{\beta}_{\mathbf{s}}\left(q^{-1}\right)$ are defined in References [22, 23]. On the other hand, the nonunique left $\tau$-inverse takes the following form $\left(N_{\mathrm{R}}>N_{\mathrm{T}}\right)$

$$
\begin{aligned}
\underline{\mathbf{C}}^{\mathrm{L}}\left(q^{-1}\right)=\{ & \mathbf{I}_{N_{\mathrm{T}}}+\left[\underline{\beta}_{\mathbf{s}}\left(q^{-1}\right)\right]_{0}^{\mathrm{L}}\left[\underline{\mathbf{C}}\left(q^{-1}\right)\right. \\
& \left.\left.-\underline{\beta}\left(q^{-1}\right)\right]\right\}^{-1}\left[\underline{\beta}_{\mathbf{s}}\left(q^{-1}\right)\right]_{0}^{\mathrm{L}} .
\end{aligned}
$$

The aforementioned forms $\left[\underline{\beta}_{\mathbf{s}}\left(q^{-1}\right)\right]_{0}^{\mathrm{R}}$ and $\left[\underline{\beta}_{\mathbf{s}}\left(q^{-1}\right)\right]_{0}^{\mathrm{L}}$ stand for the minimum-norm right and least-squares left $T$-inverses of polynomial matrix $\underline{\beta}_{\mathbf{S}}\left(q^{-1}\right)$, respectively, while $\mathbf{I}_{N_{\mathrm{T}}}$ is the identity $N_{\mathrm{T}}$-matrix.

\section{$3.3 \sigma$-inverses}

A generalization of the polynomial $\tau$-inverses is the socalled right

$$
\begin{aligned}
\underline{\mathbf{C}}^{\mathrm{R}}\left(q^{-1}\right)=\{ & \mathbf{I}_{N_{\mathrm{T}}}+\left[\underline{\beta}\left(q^{-1}\right)\right]_{0}^{\mathrm{R}}\left[\underline{\mathbf{C}}\left(q^{-1}\right)\right. \\
& \left.\left.-\underline{\beta}\left(q^{-1}\right)\right]\right\}^{-1}\left[\underline{\beta}\left(q^{-1}\right)\right]_{0}^{\mathrm{R}},
\end{aligned}
$$

and left

$$
\begin{array}{r}
\underline{\mathbf{C}}^{\mathrm{L}}\left(q^{-1}\right)=\left\{\mathbf{I}_{N_{\mathrm{T}}}+\left[\underline{\beta}\left(q^{-1}\right)\right]_{0}^{\mathrm{L}}\left[\underline{\mathbf{C}}\left(q^{-1}\right)\right.\right. \\
\left.\left.-\underline{\beta}\left(q^{-1}\right)\right]\right\}^{-1}\left[\underline{\beta}\left(q^{-1}\right)\right]_{0}^{\mathrm{L}},
\end{array}
$$

non-unique $\sigma$-inverses implementing the degrees of freedom in the form of an arbitrary matrix polynomial $\beta\left(q^{-1}\right)$.

It should be emphasized that the new forms of polynomial right and left $\sigma$-inverses (including also the parameter cases) are given in References [24, 26] as follows:

$$
\begin{aligned}
& \underline{\mathbf{C}}^{\mathrm{R}}\left(q^{-1}\right)=\underline{\beta}^{\mathrm{T}}\left(q^{-1}\right)\left[\underline{\mathbf{C}}\left(q^{-1}\right) \underline{\beta}^{\mathrm{T}}\left(q^{-1}\right)\right]^{-1}, \\
& \underline{\mathbf{C}}^{\mathrm{L}}\left(q^{-1}\right)=\left[\underline{\beta}^{\mathrm{T}}\left(q^{-1}\right) \underline{\mathbf{C}}\left(q^{-1}\right)\right]^{-1} \underline{\beta}^{\mathrm{T}}\left(q^{-1}\right) .
\end{aligned}
$$

Crucial non-unique $S$-inverses are presented below. They are effectively used when designing robust communications systems.

\subsection{S-inverses}

Non-unique polynomial $S$-inverses are associated with the so-called Smith factorization of the polynomial matrix $\underline{\mathbf{C}}\left(q^{-1}\right)$ to obtain

$$
\underline{\mathbf{C}}\left(q^{-1}\right)=\underline{\mathbf{U}}\left(q^{-1}\right) \underline{\Sigma}\left(q^{-1}\right) \underline{\mathbf{V}}\left(q^{-1}\right),
$$


where $\underline{\mathbf{U}}\left(q^{-1}\right)$ and $\underline{\mathbf{V}}\left(q^{-1}\right)$ are unimodular polynomial matrices, and the unique matrix polynomial $\underline{\Sigma}\left(q^{-1}\right)$ of dimension $N_{\mathrm{R}} \times N_{\mathrm{T}}$ includes the eigenvalues of $\underline{\mathbf{C}}\left(q^{-1}\right)$. The right and left $S$-inverses are defined as

$$
\begin{aligned}
& \underline{\mathbf{C}}^{\mathrm{R}}\left(q^{-1}\right)=\underline{\mathbf{V}}^{-1}\left(q^{-1}\right) \underline{\Sigma}^{\mathrm{R}}\left(q^{-1}\right) \underline{\mathbf{U}}^{-1}\left(q^{-1}\right), \\
& \underline{\mathbf{C}}^{\mathrm{L}}\left(q^{-1}\right)=\underline{\mathbf{V}}^{-1}\left(q^{-1}\right) \underline{\Sigma}^{\mathrm{L}}\left(q^{-1}\right) \underline{\mathbf{U}}^{-1}\left(q^{-1}\right),
\end{aligned}
$$

respectively, where (non-)unique right and left inverses of the polynomial matrix $\underline{\Sigma}\left(q^{-1}\right)$ include the degrees of freedom. Note that the parameter counterpart of the $S$ inverse strictly dedicated to state-space systems has been given in Reference [25].

Remark 1 It should be noted that all of the abovementioned inverses are reduced to the regular one $\underline{\mathbf{C}}^{-1}\left(q^{-1}\right)$ in case of $N_{\mathrm{R}}=N_{\mathrm{T}}$.

4 New approach to signal reconstruction process MIMO wireless communications systems, including multiple transmitter and receiver antennas, are becoming more and more common, and they even replace traditional SISO solutions by offering high transmission/reception channel capacity improvement. Multivariable systems ensure not only an increase in capacity but also, importantly, improvement without loss of the required technological parameters of the received signal. An intriguing case here is an approach implementing different numbers of transmitting and receiving antennas. In such non-square systems, we can find the non-uniqueness of the obtained solution, which has a positive impact on the whole signal reconstruction/recovery process. By selecting appropriate degrees of freedom of inverses, we can considerably influence the robustness and energy of the received signal (in the case of the control theory see Reference [26]). In the authors' opinion, the new method can eliminate the parasitic impact of the natural environment in the context of the applied inverses of non-square polynomial matrices. Such operations directly improve the signal transmission rate while maintaining approved quality standards. Of course, the entire signal perfect reconstruction process only occurs in case of $N_{R}>N_{\mathrm{T}}$, since we have full information about the transmitted signal.

It is important that the proposed approach to perfect reconstruction of signal is based on the polynomial matrix calculus. The solutions used so far were based on the parameter matrix calculus, using unique inverses with the so-called Hermitian conjugates of certain (full rank) non-square matrices [27]. Unfortunately, this calculus does not include the aforementioned degrees of freedom, thus making it considerably more difficult to adjust to the detrimental impact of the environment on the data transmission process.
Remark 2 It should be emphasized that the unique right and left inverses including the Hermitian conjugates are not applicable in the time-domain signal perfect reconstruction approach presented here [23].

To illustrate the discussed problems, let us analyze the stochastic process of perfect reconstruction of signal and rewrite Eq. (2) to the form

$$
\mathbf{R}^{\prime}(t)=\underline{\mathbf{C}}\left(q^{-1}\right) \mathbf{S}(t)+\zeta(t),
$$

where $\zeta(t)$ is the uncorrelated zero-mean Gaussian white noise at (discrete) time $t$.

Then, let us perform the non-unique Smith factorization of $\underline{\mathbf{C}}\left(q^{-1}\right)$ and, at the same time, eliminate ICI and ISI parasitic effects

$$
\mathbf{R}^{\prime}(t)=\underline{\mathbf{U}}\left(q^{-1}\right) \underline{\Sigma}\left(q^{-1}\right) \underline{\mathbf{V}}\left(q^{-1}\right) \mathbf{S}(t)+\zeta(t),
$$

where the polynomial matrices $\underline{\mathbf{U}}\left(q^{-1}\right)$ and $\underline{\mathbf{V}}\left(q^{-1}\right)$ are the "equalizer" and the "precoder," respectively [22].

After using the $S$-inverse, the perfect reconstruction of signal for the selected $N_{\mathrm{R}}>N_{\mathrm{T}}$ takes the following form

$$
\begin{aligned}
\mathbf{S}(t)= & \underline{\mathbf{V}}^{-1}\left(q^{-1}\right) \underline{\Sigma}^{\mathrm{L}}\left(q^{-1}\right) \underline{\mathbf{U}}^{-1}\left(q^{-1}\right) \mathbf{R}^{\prime}(t) \\
& -\underline{\mathbf{V}}^{-1}\left(q^{-1}\right) \underline{\Sigma}^{\mathrm{L}}\left(q^{-1}\right) \underline{\mathbf{U}}^{-1}\left(q^{-1}\right) \zeta(t),
\end{aligned}
$$

where the symbol "L" stands for the (non-)unique left inverse of matrix polynomial $\underline{\Sigma}\left(q^{-1}\right)$.

Of course, Eq. (16) can be rewritten in the following form

$$
\mathbf{S}^{\prime}(t)=\underline{\mathbf{V}}^{-1}\left(q^{-1}\right) \underline{\Sigma}^{\mathrm{L}}\left(q^{-1}\right) \underline{\mathbf{U}}^{-1}\left(q^{-1}\right) \mathbf{R}^{\prime}(t),
$$

with $\mathbf{S}^{\prime}(t)=\mathbf{S}(t)+\underline{\mathbf{V}}^{-1}\left(q^{-1}\right) \underline{\Sigma}^{\mathrm{L}}\left(q^{-1}\right) \underline{\mathbf{U}}^{-1}\left(q^{-1}\right) \zeta(t)$ being a stochastic $N_{\mathrm{T}}$-input vector.

Taking into account the above considerations, for $N_{\mathrm{R}}>$ $N_{\mathrm{T}}$, we obtain [28]

$\underline{\Sigma}^{\mathrm{L}}\left(q^{-1}\right)=\left[\underline{\mathbf{D}}_{N_{\mathrm{T}} \times N_{\mathrm{T}}}\left(q^{-1}\right) \underline{\mathbf{M}}_{N_{\mathrm{T}} \times\left(N_{\mathrm{R}}-N_{\mathrm{T}}\right)}\left(q^{-1}\right)\right]$,

where the polynomial matrices $\underline{\mathbf{M}}\left(q^{-1}\right)$ and $\underline{\mathbf{D}}\left(q^{-1}\right)$ include significant degrees of freedom and transmission zeros (if any in the $\underline{\mathbf{C}}\left(q^{-1}\right)$ [23]), respectively. In case of absence of transmission zeros, we have $\underline{\mathbf{D}}\left(q^{-1}\right)=\mathbf{I}_{N_{\mathrm{T}}}$.

Finally, based on the pilot knowledge, the optimal degrees of freedom of $\underline{\mathbf{M}}\left(q^{-1}\right)$ are chosen according to the square performance index

$$
\underline{\mathbf{M}}_{\mathbf{o p t}}\left(q^{-1}\right)=\underset{\underline{\mathbf{M}}\left(q^{-1}\right)}{\arg \sum_{t=0}^{N-1}}\left\{\left[\mathbf{S}^{\prime}(t)-\mathbf{S}(t)\right]^{\mathrm{T}}\left[\mathbf{S}^{\prime}(t)-\mathbf{S}(t)\right]\right\},
$$

where $N$ denotes the number of samples. 
Remark $3 \underline{\Sigma}^{\mathrm{L}}\left(q^{-1}\right)$ can also be obtained by using polynomial $\sigma$-inverses in two parallel forms

$$
\begin{gathered}
\underline{\Sigma}^{\mathrm{L}}\left(q^{-1}\right)=\left\{\mathbf{I}_{N_{\mathrm{T}}}+\left[\underline{\beta}\left(q^{-1}\right)\right]_{0}^{\mathrm{L}}\left[\underline{\Sigma}\left(q^{-1}\right)\right.\right. \\
\left.\left.-\underline{\beta}\left(q^{-1}\right)\right]\right\}^{-1}\left[\underline{\beta}\left(q^{-1}\right)\right]_{0}^{\mathrm{L}}, \\
\underline{\Sigma}^{\mathrm{L}}\left(q^{-1}\right)=\left[\underline{\beta}^{\mathrm{T}}\left(q^{-1}\right) \underline{\Sigma}\left(q^{-1}\right)\right]^{-1} \underline{\beta}^{\mathrm{T}}\left(q^{-1}\right),
\end{gathered}
$$

where $\left[\underline{\beta}\left(q^{-1}\right)\right]_{0}^{\mathrm{L}}=\left[\underline{\beta}^{\mathrm{T}}\left(q^{-1}\right) \underline{\beta}\left(q^{-1}\right)\right]^{-1} \underline{\beta}^{\mathrm{T}}\left(q^{-1}\right)$ while $\underline{\beta}\left(q^{-1}\right)$ includes the degrees of freedom [24].

Remark 4 If we apply the unique left T-inverse directly to $\underline{\mathrm{C}}\left(q^{-1}\right)$ or $\underline{\Sigma}\left(q^{-1}\right)$, we will obtain no degrees of freedom.

Remark 5 It should be pointed out that in square systems, i.e., systems having equal numbers of transmitting and receiving antennas, there are also no degrees of freedom. Therefore, the optimization cannot efficiently eliminate the impact of noise on the whole signal reconstruction process.

Remark 6 We start our optimization task in the Matlab environment with the degrees of freedom included in the parameter matrix; a more general case is based on the matrix polynomial.

Remark 7 Now, it is clear that in the deterministic case, we immediately obtain the perfect reconstruction of signal according to the following formula

$$
\mathbf{S}(t)=\underline{\mathbf{C}}^{\mathrm{L}}\left(q^{-1}\right) \mathbf{R}(t)
$$

where $\underline{\mathrm{C}}^{\mathrm{L}}\left(q^{-1}\right)$ denotes the abovementioned polynomial matrix S-inverse of full normal rank $\underline{\mathbf{C}}\left(q^{-1}\right)$.

Remark 8 It should be emphasized that in the deterministic case of the signal perfect reconstruction as mentioned in Remark 7, the left inverse of $\underline{\mathbf{C}}\left(q^{-1}\right)$ is not determined; due to the elimination of ISI and ICI drawbacks, the Sinverse has been applied. Therefore, assuming that $\mathbf{R}(t)=$ $\underline{\mathbf{C}}\left(q^{-1}\right) \mathbf{S}(t)$, the stochastic recovery task as presented in Eq. (16) can be rewritten in the following form

$$
\begin{aligned}
\mathbf{S}(t) & +\underline{\mathbf{V}}^{-1}\left(q^{-1}\right) \underline{\Sigma}^{L 1}\left(q^{-1}\right) \underline{\mathbf{U}}^{-1}\left(q^{-1}\right) \zeta(t) \\
& =\underline{\mathbf{V}}^{-1}\left(q^{-1}\right) \underline{\Sigma}^{\mathrm{L}}\left(q^{-1}\right) \underline{\mathbf{U}}^{-1}\left(q^{-1}\right) \mathbf{R}(t) \\
& +\underline{\mathbf{V}}^{-1}\left(q^{-1}\right) \underline{\Sigma}^{L 2}\left(q^{-1}\right) \underline{\mathbf{U}}^{-1}\left(q^{-1}\right) \zeta(t),
\end{aligned}
$$

where $\underline{\Sigma}^{L 1}\left(q^{-1}\right) \neq \underline{\Sigma}^{L 2}\left(q^{-1}\right)$, in general, under any $\underline{\Sigma}^{\mathrm{L}}\left(q^{-1}\right)$.

Remark 9 Since our new polynomial method of perfect signal reconstruction does not correspond to the MoorePenrose inverse, we must consider them separately.
Remark 10 The aforementioned signal recovery may be impossible after using the unique T-inverses in case of non-square systems (previously known as minimum-norm right/least-squares left inverses); these inverses may significantly destabilize the whole signal reconstruction process due to the existence of so-called unstable control zeros [22]. The whole signal reconstruction process is always destabilized in case of the existence of unstable transmission zeros which are the modes of the fundamental system matrix $\underline{\mathbf{C}}\left(q^{-1}\right)[22,23]$.

Remark 11 Note that the entire task of signal reconstruction should be understood in terms of an adaptive process, where the said degrees of freedom are selected cyclically with a period adjusted by the designer.

What is important is that the solution based on the polynomial matrix calculus (along with non-zero degrees of freedom, i.e., at $\left.\underline{\mathbf{M}}\left(q^{-1}\right) \neq \mathbf{0}\right)$, is a new approach so far unknown in the field of the modern signal reconstruction. The application of left inverses in Eq. (16) improves the capacity/robustness of the wireless communications network in terms of the elimination of the parasitic impact of noise. Of course, it is possible by choosing appropriate components/degrees of freedom of matrix $\underline{\mathbf{M}}\left(q^{-1}\right)$ of Eq. (18) according to the criterion (19). The same can be achieved as a result of applying non-unique type $\tau$ - and $\sigma$ inverses. An adequate selection of the degrees of freedom $\underline{\beta}_{\mathbf{s}}\left(q^{-1}\right)$ and $\underline{\beta}\left(q^{-1}\right)$ that are not relative to the propagation environment $\underline{\mathbf{C}}\left(q^{-1}\right)$ can provide a greater degree of independence of the parasitic effects (see Eqs. (20) and (21)). Finally, we can strongly note that an alternative to the applied Smith decomposition can be offered by the use of the PSVD method implementing the non-zero degrees of freedom, whose derivation is either based on the use of the PEVD (polynomial EVD) approach [29, 30], or one that is obtained in a direct manner [31]. This intriguing proposal is briefly described in the next section.

\section{PSVD vs. Smith decomposition-based approach}

In Reference [16], the authors applied a successful polynomial singular value decomposition. As stated earlier, the current methods in the wireless telecommunications studies use zero degrees of freedom. Hence, the idea of the use of a new non-zero degrees of freedom was conceived with the purpose of limiting the impact of the noise on the process of data transfer. The same paradigm can be applied in the signal reconstruction process based on the PSVD method, which is worth further research. However, the methods basing on SVD and PSVD decompositions cannot be directly compared with the new method applied for signal recovery. The reason for this can be associated with different dynamic parameters of the propagation environments derived as a result of using SVD and PSVD 
on one hand and a method applying polynomial $S$-inverse on the other hand. In the former case, we merely obtain an approximation of the dynamic properties of the propagation environment, whereas the latter approach implies that an accurate dynamics of this environment is obtained. Appendix offers an outline of the working characteristics of the signal reconstruction methods for a non-square system comprising two transmitting antennas and four receiving ones.

The paradigm of the new intriguing method is analytically confirmed by a more general first polynomial part of the next section containing simulation examples, whereas the second parameter part contains the results of complex optimization runs using the genetic algorithm mechanism.

\section{Results and discussion}

Let us analyze the wireless telecommunications system with no transmission zeros, including two transmitter antennas $N_{\mathrm{T}}=2$ and three receiver antennas $N_{\mathrm{R}}=3$. Assuming that the matrix obtained by the pilot identification and describing the dynamics of the parasitic impact of the environment on the signal reconstruction process takes the following form

$$
\underline{\mathbf{C}}\left(q^{-1}\right)=\left[\begin{array}{ll}
-0.3+0.5 q^{-1} & 0.6 \\
1-0.8 q^{-1}+0.4 q^{-2} & 1-0.5 q^{-1} \\
0.9-0.3 q^{-1} & 0.8
\end{array}\right] .
$$

After using the Smith factorization of $\underline{\mathbf{C}}\left(q^{-1}\right)$, we obtain

$$
\begin{gathered}
\underline{\mathbf{U}}\left(q^{-1}\right)=\left[\begin{array}{ccc}
-0.8 & 2.2-1.6 q^{-1} & \ldots \\
-1.3-0.6 q^{-1} & 0.3 & -0.3 q^{-1}-0.5 q^{-2} \\
-1 & 0 & \ldots \\
\ldots & 2.2-1.6 q^{-1} \\
\ldots & 0.6-0.3 q^{-1} \\
\ldots & 0
\end{array}\right], \\
\underline{\mathbf{V}}\left(q^{-1}\right)=\left[\begin{array}{ll}
-0.9+0.3 q^{-1} & -0.8 \\
-0.4 & 0
\end{array}\right],
\end{gathered}
$$

and containing no transmission zeros

$$
\underline{\Sigma}\left(q^{-1}\right)=\left[\begin{array}{ll}
1 & 0 \\
0 & 1 \\
0 & 0
\end{array}\right] .
$$

Now, in accordance with the signal perfect reconstruction as presented in Eq. (16), for the received signal $\mathbf{R}^{\prime}(t)=\left[R_{1}^{\prime}(t) R_{2}^{\prime}(t) R_{3}^{\prime}(t)\right]^{\mathrm{T}}$ (due to its complexity, vector $\mathbf{R}^{\prime}(t)$ blurred by a zero-mean white noise $\zeta(t)$ is not given here), we obtain the vector of the transmitted signal

$$
\begin{aligned}
& \mathbf{S}(t)=\left[\begin{array}{cc}
0 & -2.3 \\
-1.3 & 2.5-0.8 q^{-1}
\end{array}\right]\left[\begin{array}{lll}
1 & 0 & \underline{M}_{1}\left(q^{-1}\right) \\
0 & 1 & \underline{M}_{2}\left(q^{-1}\right)
\end{array}\right] \\
& \times\left[\begin{array}{ccc}
0 & 0 & \ldots \\
0.6+0.3 q^{-1} & -1 & \ldots \\
0.3-0.3 q^{-1}-0.5 q^{-2} & -2.2+1.6 q^{-1} & \ldots
\end{array}\right. \\
& \left.\begin{array}{lc}
\ldots & -1 \\
\ldots & 0.8-0.8 q^{-1} \\
\ldots & 2.5-3.2 q^{-1}+1.4 q^{-2}
\end{array}\right]\left[\begin{array}{l}
R_{1}^{\prime} \\
R_{2}^{\prime} \\
R_{3}^{\prime}
\end{array}\right] \\
& -\left[\begin{array}{cc}
0 & -2.3 \\
-1.3 & 2.5-0.8 q^{-1}
\end{array}\right]\left[\begin{array}{lll}
1 & 0 & \underline{M}_{1}\left(q^{-1}\right) \\
0 & 1 & \underline{M}_{2}\left(q^{-1}\right)
\end{array}\right] \\
& \times\left[\begin{array}{ccc}
0 & 0 & \ldots \\
0.6+0.3 q^{-1} & -1 & \ldots \\
0.3-0.3 q^{-1}-0.5 q^{-2} & -2.2+1.6 q^{-1} & \ldots
\end{array}\right. \\
& \left.\begin{array}{lc}
\ldots & -1 \\
\ldots & 0.8-0.8 q^{-1} \\
\ldots & 2.5-3.2 q^{-1}+1.4 q^{-2}
\end{array}\right] \zeta(t) .
\end{aligned}
$$

Finally, for the determined value $\zeta(t)=\left[\begin{array}{ll}0.1 & -\end{array}\right.$

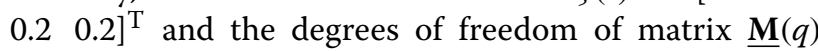
$=\left[\frac{343712}{841\left(1996 q^{2}-2023 q+521\right)} \frac{14944\left(-38348 q^{2}+15109 q\right)}{707281\left(1996 q^{2}-2023 q+521\right)}\right]=$ $\left[\underline{M}_{1}(q) \underline{M}_{2}(q)\right]^{\mathrm{T}}$, selected as a result of analytical calculations, the reconstructed vector $\mathbf{S}(t)$ is $\mathbf{S}(t)=[3+3 i-$ $3-i]^{\mathrm{T}}$ (corresponding to the two points of 16-QAM constellation of transmitted signal $\mathbf{S}(t))$. Note that in our simulation example, there is $\underline{\Sigma}^{\mathrm{L}}\left(q^{-1}\right)=\underline{\Sigma}^{\mathrm{L} 1}\left(q^{-1}\right)=$ $\underline{\Sigma}^{\mathrm{L} 2}\left(q^{-1}\right)$, see Remark 8 .

To better describe the advantages of the method proposed, complex tests were carried out by using the authors' OFDM technology simulator running in the Matlab environment [28]. For this purpose, 103776 bits of random input data from 64-QAM constellation were transferred by means of the IQ-modulated signal through the single carrier system with the matrix $\underline{\mathbf{C}}\left(q^{-1}\right)$ as in Eq. (24). For the assumed rigorous tolerance, the special parameter matrices $\underline{\mathbf{M}}\left(q^{-1}\right)$ were obtained using the genetic algorithm according to the performance index (19). Thus, we have different degrees of freedom for each of the SNRs, not presented in this paper due to space limitation. It is evident that the new method outperforms the classical one, where zero degrees of freedom associated with the application of minimum-norm/least-squares inverses to $\underline{\Sigma}\left(q^{-1}\right)$ a polynomial matrix can be find. This statement is confirmed in Fig. 1.

In addition, two simulation tests were performed covering propagation environments described by the following matrices

$\underline{\mathbf{C}}\left(q^{-1}\right)=\left[\begin{array}{cc}-0.75+0.6 q^{-1} & 0.9 \\ 0.56-0.01 q^{-1}+0.02 q^{-2} & 0.83-0.75 q^{-1} \\ 0.83-0.25 q^{-1} & 0.6\end{array}\right]$, 


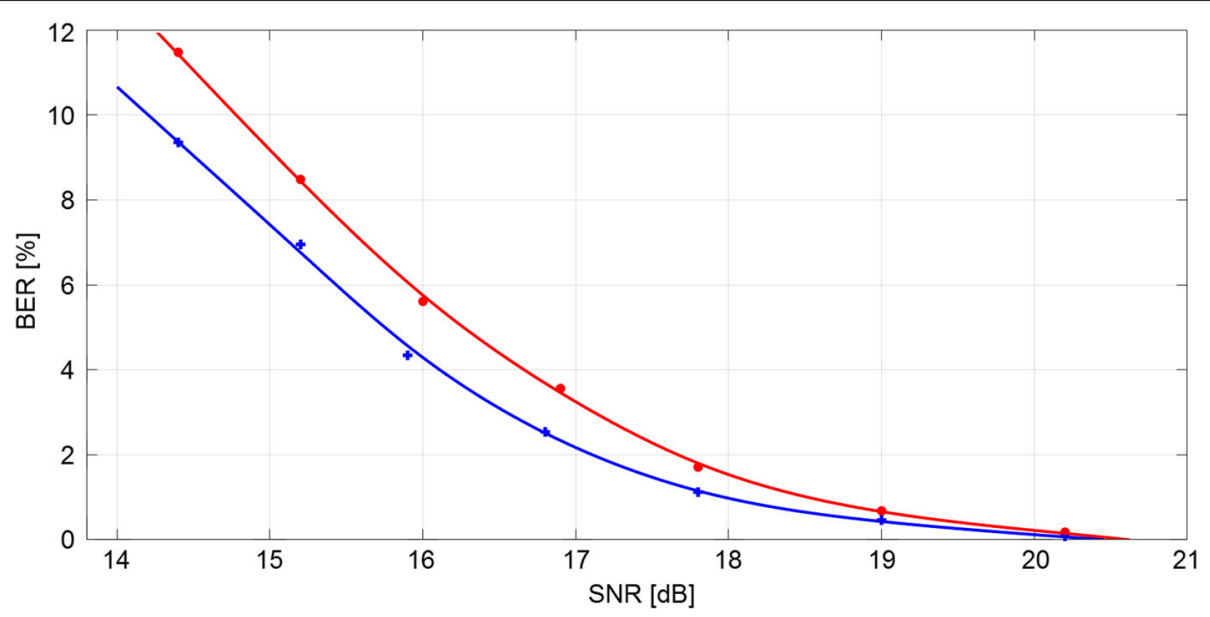

Fig. 1 Perfect reconstruction process: BER vs. SNR

and

$$
\underline{\mathbf{C}}\left(q^{-1}\right)=\left[\begin{array}{cc}
-0.5+0.4 q^{-1} & 0.9 \\
1-0.6 q^{-1}+0.2 q^{-2} & 1-0.6 q^{-1} \\
1-0.4 q^{-1} & 0.6
\end{array}\right],
$$

respectively.

Figure 2 presents the results obtained for single carrier system given by Eq. (29), whereas Fig. 3 for single carrier system as in Eq. (30).

\section{Conclusions}

In this paper, the new approach to the process of perfect reconstruction of signals is presented. The new solution is based on polynomial matrix calculus, mainly the so-called left $S$-inverse of the polynomial matrix. Errors generated in the process of signal reconstruction are compensated by the appropriate selection of components/degrees of freedom of the non-zero matrix
$\underline{\mathbf{M}}\left(q^{-1}\right)$. Simulation tests carried out in the Matlab environment have indicated a considerable implementation potential of the innovative approach proposed in this paper to the tasks of efficient signal recovery in nonsquare MIMO telecommunications systems. It should be emphasized that the new method still outperforms the typical one in case of presence of noise with uniform distribution.

\section{Appendix}

\section{Method based on PSVD}

The matrix applied to described the dynamics of the propagation environment assumes the form

$$
\underline{\mathbf{C}}\left(q^{-1}\right)=\left[\begin{array}{lr}
1.5 q^{-1} & -3 \\
-3 q^{-2} & -1.5 q^{-1} \\
1.5 q^{-1} & -3 \\
-3 q^{-2} & -1.5 q^{-1}
\end{array}\right]
$$

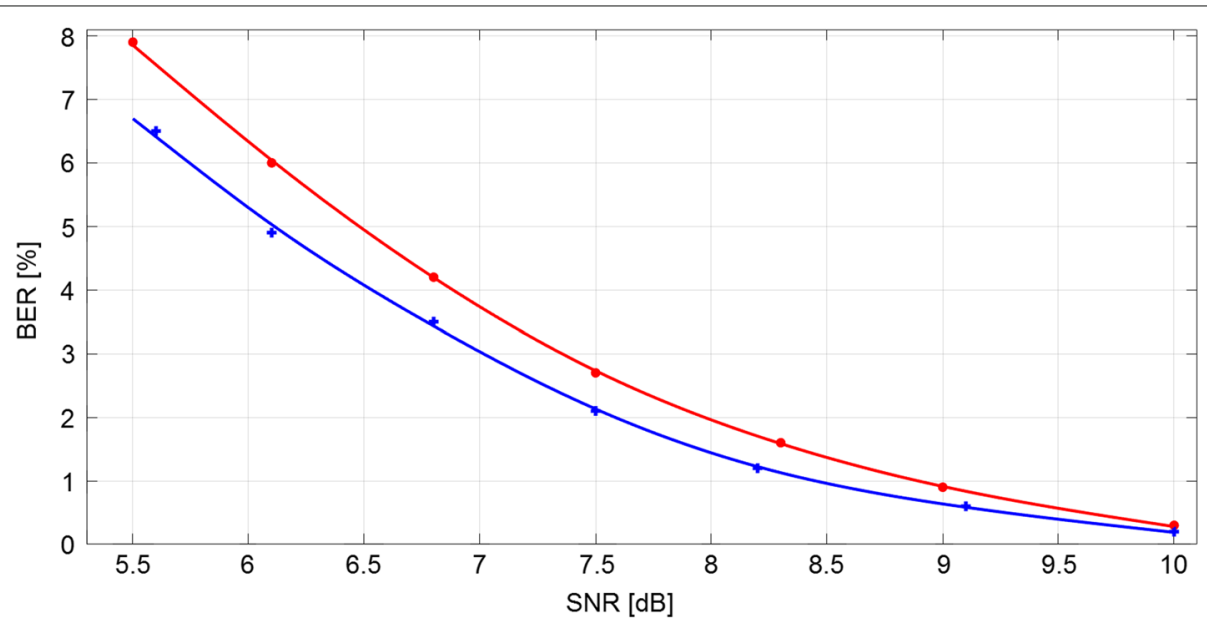

Fig. 2 Perfect reconstruction process: BER vs. SNR 


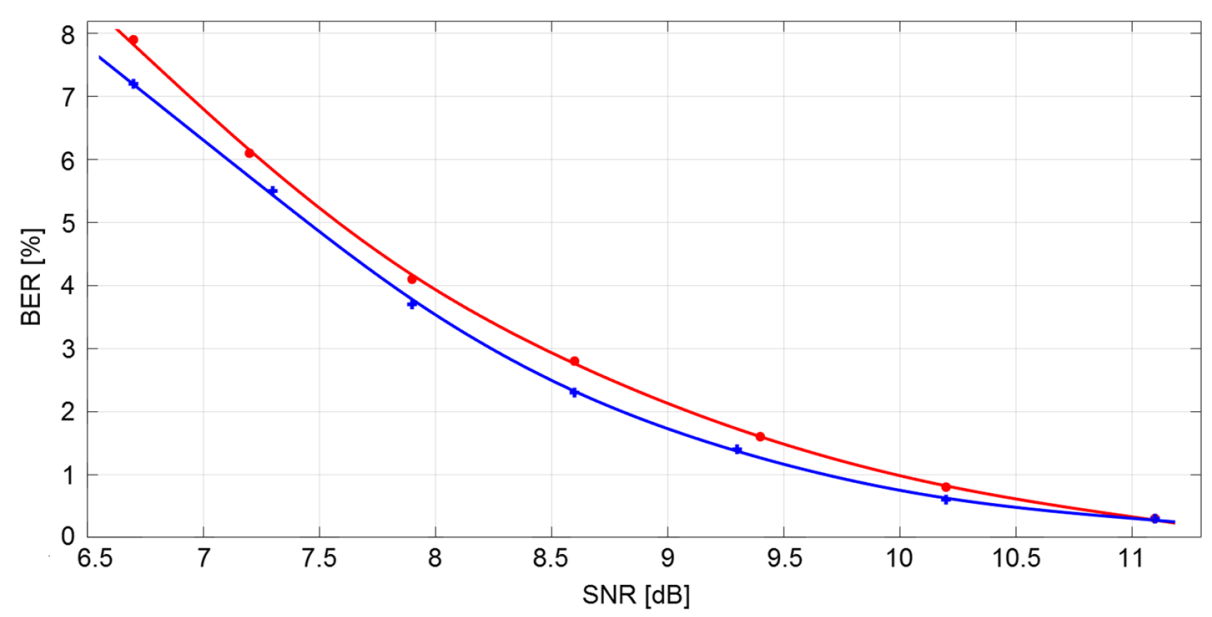

Fig. 3 Perfect reconstruction process: BER vs. SNR

Following PSVD factorization, we receive

$$
\underline{\mathbf{C}}\left(q^{-1}\right)=\mathbf{U}(q) \Sigma(q) \mathbf{V}^{\dagger}(q) \text {, }
$$

where

$$
\mathbf{U}(q)=\left[\begin{array}{lrrr}
0.5 & q & 0.5 & q^{2} \\
-q^{-1} & 0.5 & -q^{-2} & 0.5 \\
0.5 & q & -0.5 & -q^{2} \\
-q^{-1} & 0.5 & q^{-2} & -0.5
\end{array}\right], \Sigma(q)=\left[\begin{array}{ll}
q & 0 \\
0 & 1 \\
0 & 0 \\
0 & 0
\end{array}\right]
$$

and $\mathbf{V}^{\dagger}(q)=\mathbf{V}^{\mathrm{H}}\left(1 / q^{*}\right)=\left[\begin{array}{cc}3 q^{-2} & 0 \\ 0 & -3 q^{-1}\end{array}\right]$.

Evidently, $\mathbf{U}(q) \mathbf{U}^{\dagger}(q)=\mathbf{U}^{\dagger}(q) \mathbf{U}(q)=2.5 \mathbf{I}_{4}$ and $\mathbf{V}(q)$ $\mathbf{V}^{\dagger}(q)=\mathbf{V}^{\dagger}(q) \mathbf{V}(q)=9 \mathbf{I}_{2}$, where $\mathbf{I}_{n}$ denotes $n$-identity matrix, and both $\mathbf{U}(q)$ and $\mathbf{V}(q)$ are paraunitary matrices.

Let us consider a deterministic process of perfect signal reconstruction

$$
\mathbf{R}(t)=\underline{\mathbf{C}}\left(q^{-1}\right) \mathbf{S}(t),
$$

where $\mathbf{R}(t)$ and $\mathbf{S}(t)$ are the vectors of the received and transmitted signals, respectively. By consideration of Eq. (I.2) and application of the precoder $\mathbf{V}(q)$ and equalizer $\mathbf{U}^{\dagger}(q)$ structures (for an example see Reference [15]), we receive

$$
\mathbf{R}^{\prime}(t)=\mathbf{U}^{\dagger}(q) \mathbf{U}(q) \Sigma(q) \mathbf{V}^{\dagger}(q) \mathbf{V}(q) \mathbf{S}(t) .
$$

Unfortunately, $\mathbf{R}^{\prime}(t) \neq \mathbf{R}(t)$.

\section{Method based on SVD}

By the analogy to the case of PSVD for $\mathbf{R}(t)=\mathbf{C S}(t)$, where $\mathbf{C}$ is a parameter matrix, we receive

$$
\mathbf{R}^{\prime}(t)=\mathbf{U}^{\dagger} \mathbf{U} \Sigma \mathbf{V}^{\dagger} \mathbf{V S}(t)
$$

where $\mathbf{V}$ and $\mathbf{U}^{\dagger}$ denote the precoder and equalizer structures, respectively, fulfilling the condition of unitarity.

In this case, also $\mathbf{R}^{\prime}(t) \neq \mathbf{R}(t)$.

\section{Method based on polynomial S-inverse}

Taking into consideration that

$$
\underline{\mathbf{C}}\left(q^{-1}\right)=\underline{\mathbf{U}}\left(q^{-1}\right) \underline{\Sigma}\left(q^{-1}\right) \underline{\mathbf{V}}\left(q^{-1}\right),
$$

where $\underline{\mathbf{U}}\left(q^{-1}\right)$ and $\underline{\mathbf{V}}\left(q^{-1}\right)$ are unimodular matrices obtained as a result of applying Smith factorization, Eq. (I.3) can be written in the following form

$$
\mathbf{R}(t)=\underline{\mathbf{U}}\left(q^{-1}\right) \underline{\Sigma}\left(q^{-1}\right) \underline{\mathbf{V}}\left(q^{-1}\right) \mathbf{S}(t) .
$$

By solving Eq. (III.7) in respect to $\mathbf{S}(t)$, we receive the actual error-free transmitted signal $\mathbf{S}(t)$ in accordance with the relation

$$
\mathbf{S}(t)=\underline{\mathbf{V}}^{-1}\left(q^{-1}\right) \underline{\Sigma}^{\mathrm{L}}\left(q^{-1}\right) \underline{\mathbf{U}}^{-1}\left(q^{-1}\right) \mathbf{R}(t),
$$

where superscript "L" denotes every non-unique left inverse of matrix polynomial $\underline{\Sigma}^{\mathrm{L}}\left(q^{-1}\right)$.

Finally, let us remark that we cannot directly compare the two methods of signal recovery, i.e., approaches involving respective (P)SVD and Smith factorization mechanisms. The reason for this was associated, for example, with the lack of causation phenomenon in the precoder and/or equalizer structures (see Eq. (I.4), where, e.g., $\mathbf{U}(q)=\left[\begin{array}{lrrr}0.5 & q & 0.5 & q^{2} \\ -q^{-1} & 0.5 & -q^{-2} & 0.5 \\ 0.5 & q & -0.5 & -q^{2} \\ -q^{-1} & 0.5 & q^{-2} & -0.5\end{array}\right]$, whereas for the case when, e.g., $q^{2}$ constitutes a double feed-forward, i.e., we have $y(t)=u(t+2))$.

\section{Abbreviations}

BER: Bit error rate; FIR: Finite impulse response; ICl: Inter-channel interference; ISI: Inter-symbol interference; MIMO: Multi-input/multi-output; OFDM: Orthogonal frequency division multiplexing; PEVD: Polynomial eigenvalue decomposition; PSVD: Polynomial singular value decomposition; SISO: Single-input/single-output; SNR: Signal-to-noise ratio

\section{Acknowledgements}

Invaluable comments from the anonymous reviewers are gratefully acknowledged. 


\section{Funding}

This work was funded by the Department of Electrical, Control and Computer Engineering, Opole University of Technology, Poland.

\section{Competing interests}

Both authors declare that they have no competing interests.

\section{Authors' contributions}

Both authors contributed to the work. Both authors read and approved the final manuscript.

\section{Publisher's Note}

Springer Nature remains neutral with regard to jurisdictional claims in published maps and institutional affiliations.

Received: 30 May 2017 Accepted: 20 April 2018

Published online: 08 May 2018

\section{References}

1. A Goldsmith, SA Jafar, N Jindal, S Vishwanath, Capacity limits of MIMO channels. IEEE J. Sel. Areas Commun. 21(5), 684-702 (2003)

2. HShu, EP Simon, L Ros, On the use of tracking loops for low-complexity multi-path channel estimation in OFDM systems. Signal Process. 117, 174-187 (2015)

3. Z Zhao, M Schellmann, X Gong, Q Wang, R Böhnke, Y Guo, Pulse shaping design for OFDM systems. EURASIP J. Wirel. Commun. Netw. 2017(74) (2017). https://jwcn-eurasipjournals.springeropen.com/track/pdf/10. 1186/s13638-017-0849-8

4. X Ji, Z Bao, Ch Xu, Power minimization for OFDM modulated two-way amplify-and-forward relay wireless sensor networks. EURASIP J. Wirel. Commun. Netw. 2017(70) (2017). https://jwcn-eurasipjournals. springeropen.com/track/pdf/10.1186/s13638-017-0848-9

5. D Mattera, M Tanda, Optimum single-tap per-subcarrier equalization for OFDM/OQAM systems. Digit. Signal Process. 49, 148-161 (2016)

6. M Nagahara, Discrete signal reconstruction by sum of absolute values. IEEE Signal Process. Lett. 22(10), 1575-1579 (2015)

7. O Nibouche, J Jiang, Palmprint matching using feature points and SVD factorisation. Digit. Signal Process. 23(4), 1154-1162 (2013)

8. QH Spencer, A Lee Swindlehurst, M Haardt, Zero-forcing methods for downlink spatial multiplexing in multiuser MIMO channels. IEEE Trans. Signal Process. 52(2), 461-471 (2004)

9. EKS Au, S Jin, MR McKay, WH Mo, Analytical performance of MIMO-SVD systems in ricean fading channels with channel estimation error and feedback delay. IEEE Trans. Wirel. Commun. 7(4), 1315-1325 (2008)

10. D Sellathambi, J Srinivasan, S Rajaram, in Proc. of the International Conference on Design and Manufacturing (IConDM'2013), Procedia Engineering, 64. Hardware implementation of adaptive SVD beamforming algorithm for MIMO-OFDM systems (Elsevier, Chennai, 2013), pp. 84-93

11. M Sadek, A Tarighat, AH Sayed, A leakage-based precoding scheme for downlink multi-user MIMO channels. IEEE Trans. Wirel. Commun. 6(5), 1711-1721 (2007)

12. W-Q Wang, H Shao, J Cai, MIMO antenna array design with polynomial factorization. International Journal of Antennas and Propagation, Special Issue: MIMO Antenna Design and Channel Modeling. 358413, 9 (2013). https://www.hindawi.com/journals/ijap/2013/358413/

13. J Lebrun, $P$ Comon, Blind algebraic identification of communication channels: symbolic solution algorithms. AAECC. 17(6), 471-485 (2006)

14. GH Norton, On the annihilator ideal of an inverse form. AAECC. 28(1), 31-78 (2017)

15. IA Akhlaghi, H Khoshbin, A novel method for singular value decomposition of polynomial matrices and $\mathrm{ICl}$ cancellation in a frequency-selective MIMO channel. Int. J. Tomogr. Stat. 11(S09), 83-98 (2009)

16. N Moret, A Tonello, S Weiss, in Proc. of the 73rd IEEE Vehicular Technology Conference (VTC'2011). MIMO precoding for filter bank modulation systems based on PSVD (Budapest), pp. 1-5

17. H Zamiri-Jafarian, M Rajabzadeh, in Proc. of the 67th IEEE Vehicular Technology Conference (VTC'2008). A polynomial matrix SVD approach for time domain broadband beamforming in MIMO-OFDM systems, (Marina Bay), pp. 802-806
18. L Li, G Gu, Design of optimal zero-forcing precoders for MIMO channels via optimal full information control. IEEE Trans. Signal Process. 53(8), 3238-3246 (2005)

19. S Li, J Zhang, L Chai, in Proc. of the 20th European Signal Processing Conference (EUSIPCO'2012). Noncausal zero-forcing precoding subject to individual channel power constraints, (Bucharest), pp. 1623-1627

20. A Scaglione, S Barbarossa, GB Giannakis, Filterbank transceivers optimizing information rate in block transmissions over dispersive channels. IEEE Trans. Inf. Theory. 45(3), 1019-1032 (1999)

21. AV Krishna, Hari KVS, Filter bank precoding for FIR equalization in high-rate MIMO communications. IEEE Trans. Signal Process. 54(5), 1645-1652 (2006)

22. WP Hunek, Towards a General Theory of Control Zeros for LTI MIMO Systems. (Opole University of Technology Press, Opole, 2011)

23. WP Hunek, KJ Latawiec, A study on new right/left inverses of nonsquare polynomial matrices. Int. J. Appl. Math. Comput. Sci. 21(2), 331-348 (2011)

24. WP Hunek, KJ Latawiec, R Stanisławski, M Łukaniszyn, P Dzierwa, in Proc. of the 18th IEEE International Conference on Methods and Models in Automation and Robotics (MMAR'2013). A new form of a $\sigma$-inverse for nonsquare polynomial matrices, ((Miedzyzdroje), pp. 282-286

25. WP Hunek, in Proc. of the 20th IEEE International Conference on System Theory, Control and Computing (ICSTCC'2016). New SVD-based matrix $H$-inverse vs. minimum-energy perfect control design for state-space LTI MIMO systems, (Sinaia), pp. 14-19

26. WP Hunek, in Proc. of the 8th IFAC Symposium on Robust Control Design (ROCOND'2015). An application of new polynomial matrix $\sigma$-inverse in minimum-energy design of robust minimum variance control for nonsquare LTI MIMO systems, (Bratislava), pp. 149-153

27. Y Zhang, Y Li, M Gao, Robust adaptive beamforming based on the effectiveness of reconstruction. Signal Process. 120, 572-579 (2016)

28. P Majewski, Research towards increasing the capacity of wireless data communication using inverses of nonsquare polynomial matrices. (PhD thesis, Opole University of Technology, Opole, 2017)

29. JG McWhirter, PD Baxter, T Cooper, S Redif, J Foster, An EVD algorithm for para-Hermitian polynomial matrices. IEEE Trans. Signal Process. 55(5), 2158-2169 (2007)

30. S Weiss, AP Millar, RW Stewart, in Proc. of the 18th European Signal Processing Conference (EUSIPCO'2010). Inversion of parahermitian matrices, (Aalborg), pp. 447-451

31. JG McWhirter, in Proc. of the 18th European Signal Processing Conference (EUSIPCO'2010). An algorithm for polynomial matrix SVD based on generalised Kogbetliantz transformations, (Aalborg), pp. 457-461

\section{Submit your manuscript to a SpringerOpen ${ }^{\circ}$ journal and benefit from:}

- Convenient online submission

- Rigorous peer review

- Open access: articles freely available online

- High visibility within the field

- Retaining the copyright to your article

Submit your next manuscript at $>$ springeropen.com 